\title{
Cholesterol induces autophagic and apoptotic death in gastric carcinoma cells
}

\author{
SUNG-CHUL LIM ${ }^{1,2}$, KESHAB RAJ PARAJULI ${ }^{1}$, HONG-QUAN DUONG ${ }^{1}$, \\ JEONG EUN CHOI ${ }^{1,3}$ and SONG IY HAN ${ }^{1,3}$ \\ ${ }^{1}$ Research Center for Resistant Cells, ${ }^{2}$ Department of Pathology, College of Medicine, Chosun Universtity, \\ ${ }^{3}$ BioBank, Chosun University Hospital, Gwangju 501-759, Republic of Korea
}

Received October 2, 2013; Accepted October 30, 2013

DOI: $10.3892 /$ ijo.2014.2246

\begin{abstract}
Despite conflicting results, there is evidence to suggest an inverse link between total body cholesterol levels and the risk of certain malignancies. Based on previous reports, this phenomenon appears to vary with cancer site, and, in particular, more consistent data on inverse relations was reported in the risk of gastric cancer. In the current study, the effect of cholesterol on gastric cancer cell viability was examined using an in vitro cell culture system. Addition of cholesterol in culture medium resulted in reduced viability and clonogenicity of SNU601, SNU638 and SNU216 gastric cancer cells by induction of both autophagic and apoptotic death. Transient inactivation of ERK1/2 was linked to reduction of cholesterol-mediated cell viability, and tumor necrosis factor-related apoptosis-inducing ligand receptor 2 (TRAIL-R2/DR5) was also involved in cell death signaling. In conclusion, these results imply that cholesterol can act as a signal regulator to modulate cell viability and that proper cellular cholesterol levels may be advantageous to suppress growth of gastric carcinomas.
\end{abstract}

\section{Introduction}

Gastric cancer (GC) remains one of the most frequently occurring malignancies world-wide. Various surgical and chemotherapeutic treatments have been developed (1), and surgical operation is highly effective in early-stage cancers (2). However, GC is still a formidable disease because many cases of GC are diagnosed at an advanced stage and recur even after complete resection.

The prevalence of GC is associated with several factors, including environment, diet, microbial infection and genetic background $(3,4)$. Intake of fruits, vegetables and high

Correspondence to: Dr Song Iy Han, BioBank, Chosun University Hospital, Gwangju 501-759, Republic of Korea

E-mail: sihan@chosun.ac.kr

Key words: gastric cancer, cholesterol, apoptosis, autophagic deagh, tumor necrosis factor-related apoptosis-inducing ligand receptor 2 $\beta$-carotene containing foods has been reported to reduce the risk of GC (5-7), while consumption of salted meats appears to increase the risk of the disease (8). Despite conflicting results serum cholesterol level has been suggested as one of the factors affecting the risk of GC $(9,10)$. High level of serum cholesterol has been well established as a major risk factor for coronary heart disease and stroke, and has been implicated in prostate cancer and breast cancer. In contrast, several studies of cancer epidemiology have indicated that the lowest level of total cholesterol may also be dangerous because it is associated with an increased risk of cancer mortality $(11,12)$. A number of studies have suggested an apparent inverse association between serum cholesterol level and incidence and mortality of GC (13-17). However, cellular responses induced by environmental cholesterol level have not been studied in carcinomas. In this study, a cell culture model system was used to clarify the association of cholesterol level and cell viability in GC cells.

\section{Materials and methods}

Cell culture. SNU601, SNU638 and SNU216 human GC cell lines obtained from the Korea Cell Line Bank were grown in RPMI-1640 medium (Invitrogen) supplemented with 10\% (v/v) fetal bovine serum and $1 \%$ antibiotics at $37^{\circ} \mathrm{C}$ in $5 \% \mathrm{CO}_{2}$.

Hoechst 33342 staining and monodansylcadaverine $(M D C)$ staining. Treated cells were incubated with $1 \mu \mathrm{g} / \mathrm{ml}$ Hoechst 33342 at $37^{\circ} \mathrm{C}, 5 \% \mathrm{CO}_{2}$ for $15 \mathrm{~min}$ in the dark, and both floating and attached cells were collected by centrifugation. The pooled cell pellets were washed with ice-cold phosphate-buffered saline (PBS) and fixed in 3.7\% formaldehyde on ice, then washed again with PBS, re-suspended and a fraction of the suspension was centrifuged in a cytospinner (Hanil, Korea). The slides were air dried, mounted in an anti-fade solution, and examined using a DM5000 fluorescence microscope (Leica, Germany) at respective excitation/emission wavelengths of $340 / 425 \mathrm{~nm}$. A total of 500 cells from randomly chosen fields were counted and the number of apoptotic cells was expressed as a percentage of the total number of cells counted. For staining of autophagic vacuoles, treated cells were incubated with MDC for $30 \mathrm{~min}$ and washed with PBS and observed under a fluorescence microscope at $340 / 525 \mathrm{~nm}$. 
A

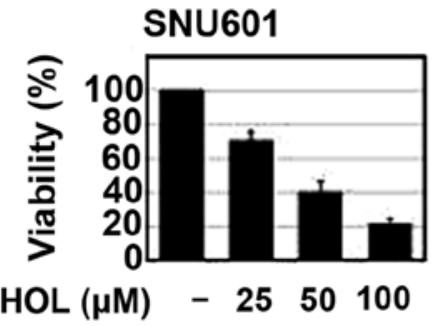

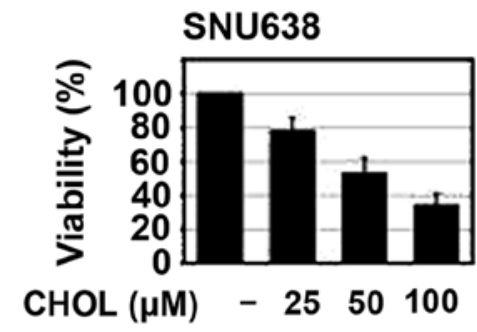

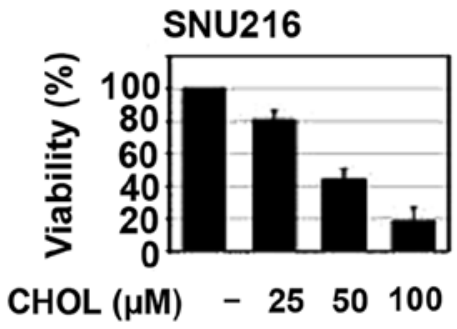

B
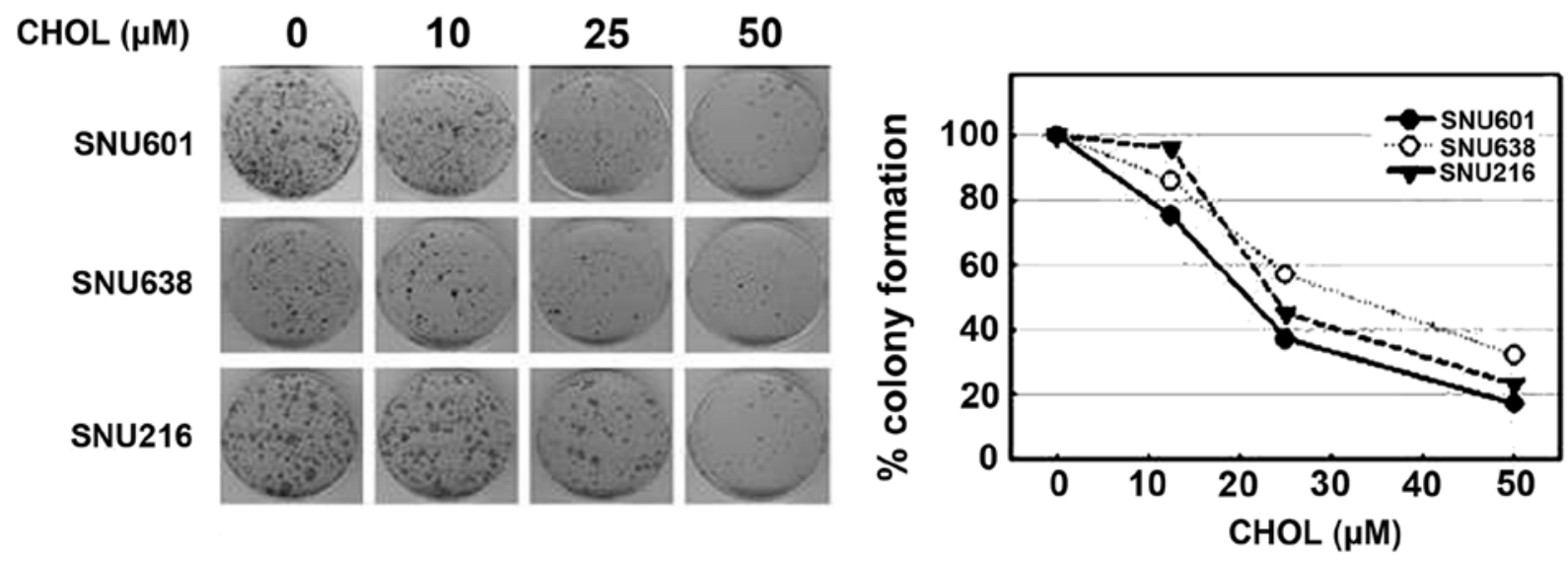

Figure 1. Cholesterol suppresses cell viability and colony forming ability in GC cells. (A) Cells were exposed to the indicated concentrations of cholesterol for $48 \mathrm{~h}$ and viability was measured by MTT assay. (B) Cells were exposed to $0,10,25$ and $50 \mu \mathrm{M}$ of cholesterol for $12 \mathrm{~h}$ and 2,000 cells were re-plated on 60 -mm dishes; colonies were counted after two weeks of incubation.

MTT assay. Cells were incubated with MTT solution $(0.5 \mathrm{mg} / \mathrm{ml})$ for $4 \mathrm{~h}$ and solubilized using dimethylsulfoxide, and the solubilized formazan product was quantified using an enzyme-linked immunosorbant assay (ELISA) plate reader at $595 \mathrm{~nm}$; absorbance of untreated cells was designated as $100 \%$ and cell survival was expressed as a percentage of this value.

Clonogenic assay. Cells treated with cholesterol for $12 \mathrm{~h}$ were trypsinized, washed and re-plated (2,000 cells/60-mm dish). After incubation for 14 days in a $37^{\circ} \mathrm{C} / 5 \% \mathrm{CO}_{2}$ incubator, colonies were stained using crystal violet and scored ( $>1 \mathrm{~mm})$.

Immunoblot analysis. Equal amounts of protein were electrophoretically separated using SDS-PAGE and transferred to a nitrocellulose membrane using a standard technique. Antibodies were used to probe for active P-ERK1/2, P-MEK1, MEK1, P-JNK1/2, P-p38, p38, caspase-3 (Cell Signaling Technology), PARP, Fas, caveolin-1, ERK2, JNK1/2, cytochrome $c$ (Santa Cruz), DR4, DR5 (Pro Sci), ATG5 and LC3II (MBL International). Anti- $\alpha$-tubulin (BioGenex) was used as a loading control. Acquisition of signals was performed using an image analyzer (Image Station 4000MM, Kodak).

Caspase activity assay. The caspase- 8 activity assay was performed using a FADD-like IL- $1 \beta$-converting enzyme (FLICE) colorimetric assay kit (BioVision), according to the manufacturer's protocol. Briefly, $200 \mu \mathrm{g}$ protein lysates in a $50-\mu 1$ volume was mixed with reaction buffer, mixed with
IETD-pNA substrate, incubated for $90 \mathrm{~min}$, and the absorbance was measured at $405 \mathrm{~nm}$. Fold increase in FLICE activity was determined by comparing the results of treated samples with the level of the untreated control.

Cell transfection. The constitutively active mutant of MEK1 (CA-MEK1) was designed by substitution of the regulatory phosphorylation sites, $\operatorname{Ser}^{218}$ and $\mathrm{Ser}^{222}$, with aspartic acid (S218D/S222D mutant), as described previously (18), and cloned into the pCMV vector. Then, cells were transfected transiently for $48 \mathrm{~h}$ and the protein amounts of MEK1 and p-ERKs were confirmed by western blot analysis. For the RNAi, siRNA were obtained from Bioneer and 5-8 $\mu \mathrm{g} / \mathrm{ml}$ RNA were used for transfection by use of AMAXA nucleofector kit (Amaxa Biosystems $\mathrm{GmbH}$ ) according to the manufacturer's protocol. Sequences of siRNA are as follows: control sense, 5'-CCUAC GCCACCAAUUUCGU(dTdT)-3'; DR5 sense, 5'-CAGACUU GGUGCCCUUUGA(dTdT)-3'.

Statistical analysis. All numerical data are reported as mean \pm SE. All data represent the results of at least three independent experiments. Groups were compared using Student's t-test.

\section{Results}

Cholesterol induces autophagic and apoptotic death. To assess the effect of cholesterol on the viability of GC cells, 
A

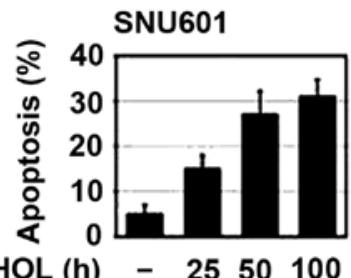

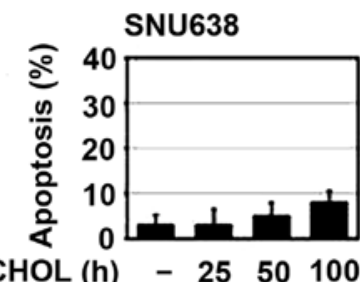

CHOL (h) - 2550100

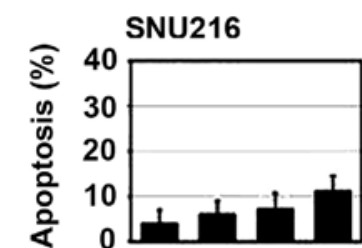

CHOL (h) - 2550100

B
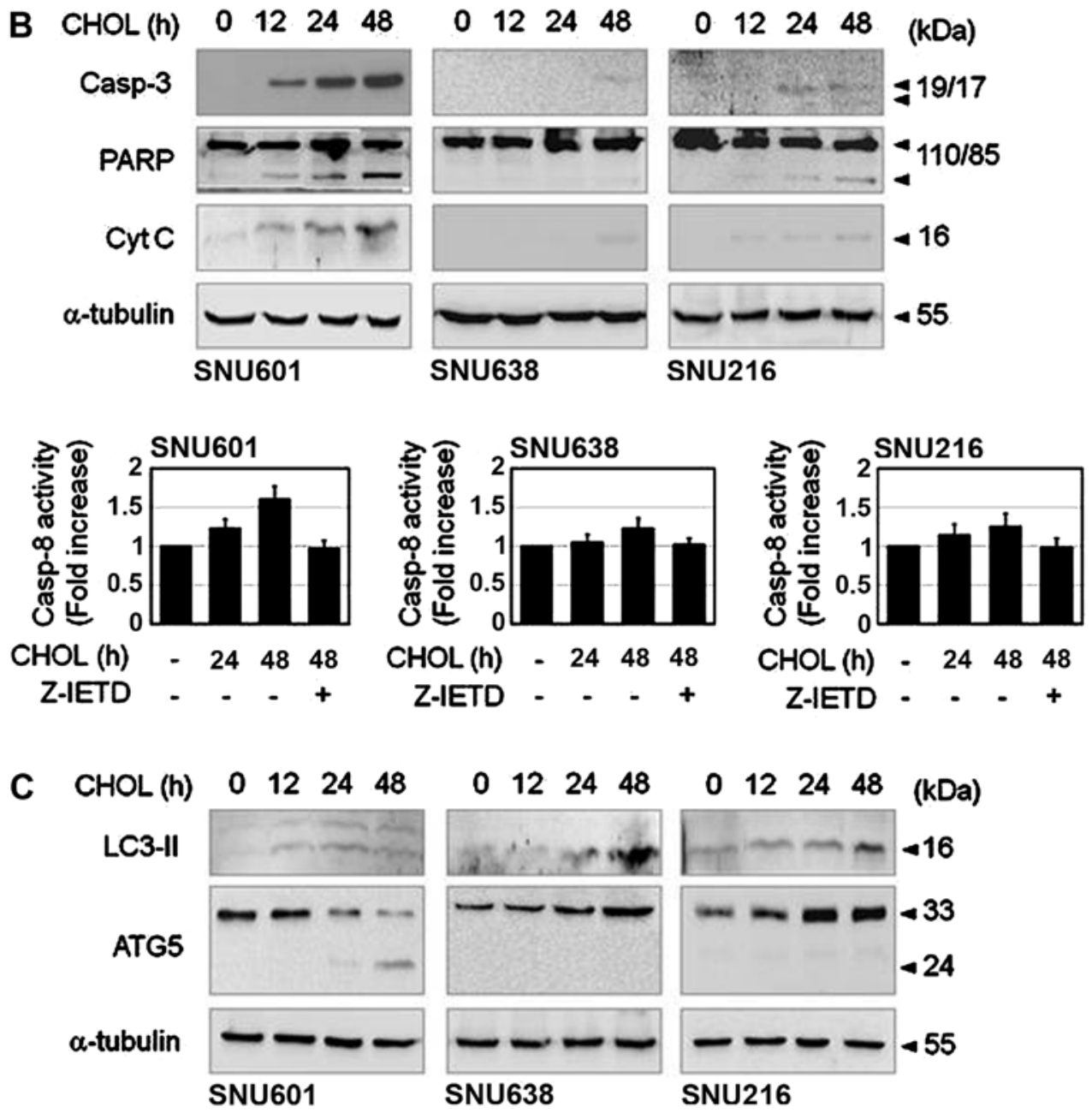

Figure 2. Cholesterol induces apoptotic and autophagic cell death in GC cells. (A) Cells untreated or treated with 25,50 and $100 \mu \mathrm{M}$ cholesterol for $48 \mathrm{~h}$ were stained with Hoechst 33342 and fragmented or condensed apoptotic cells were assessed under a fluorescence microscope. (B) Cells treated with $50 \mu \mathrm{M}$ cholesterol for the indicated times were analyzed by immunoblotting for detection of active caspase-3, PARP and released cytoplasmic cytocrome $c$ (upper panels) or subjected to caspase-8 activity assay (lower panels). (C) Cells treated with $50 \mu \mathrm{M}$ cholesterol for the indicated times were analyzed by immunoblotting for detection of LC3II and ATG5.

a number of different human stomach cancer cell lines, SNU601, SNU638 and SNU216, were used in the study. Incubation of these cells in a culture medium with addition of different concentrations of water-soluble cholesterol resulted in a dose-dependent decrease in cell viability in all three cell lines, as determined by the MTT assay. In addition, treatment with cholesterol also resulted in a marked, dose-dependent decrease in colony forming ability of these cells (Fig. 1). Thus, cholesterol may exert antitumor activity in GC cells.

To exclude the specific effect of increased water solubility of cholesterol, we tested the effect of hydrophobic cholesterol solubilized in acetic acid in these cells; water-insoluble hydrophobic cholesterol also reduced cell viability when compared with the control group, the same as water-soluble cholesterol treatment (data not shown). Then, in order to explore the cause of cholesterol-induced viability reduction, we attempted to determine whether cholesterol induces apoptosis in GC cells. Cells exposed to cholesterol were stained by Hoechst 33342 and apoptotic nuclei were then counted. Upon exposure to cholesterol, apoptotic body formation showed a clear increase in SNU601 cells, however, only a slight increase was observed in SNU638 and SNU216 cells (Fig. 2A). Cholesterol also strongly induced cleavage of caspase-3, degradation of PARP and activation of caspase-8 in SNU601 cells, and weakly in SNU638 and SNU216 cells, as demonstrated by immunoblot analysis and caspase activity assay (Fig. 2B). Although viability of all three 
A

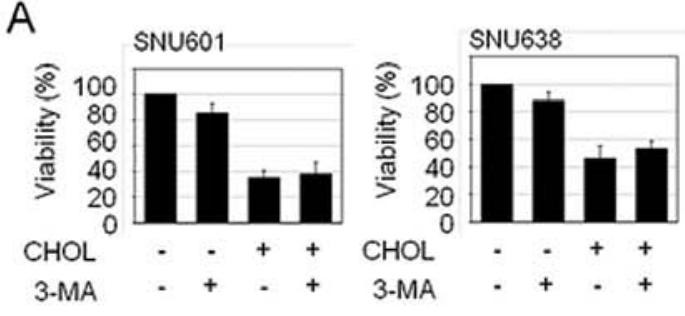

B

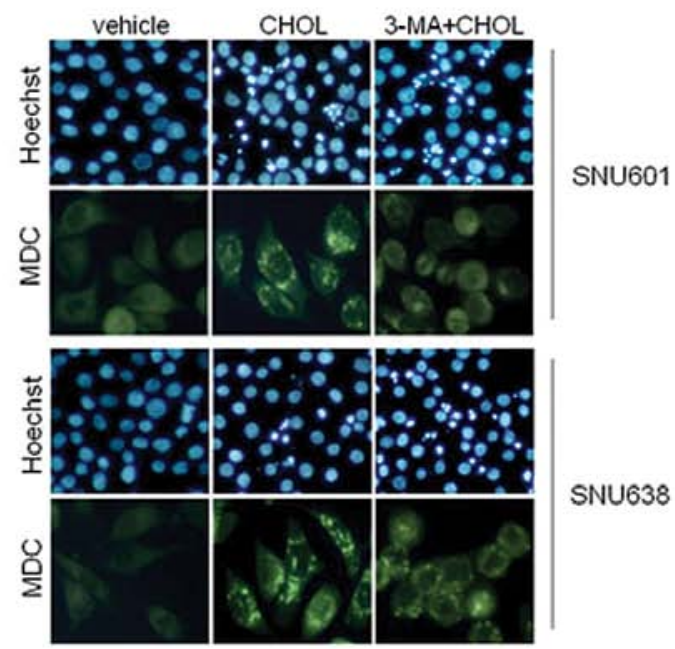

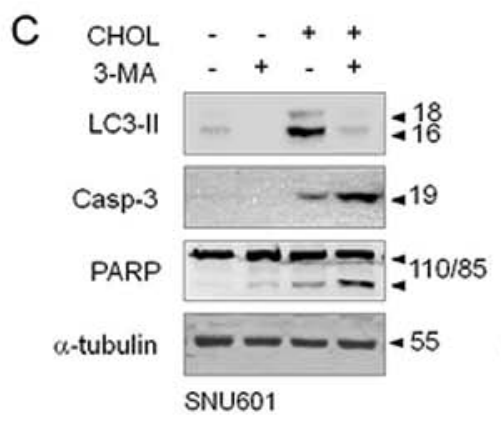

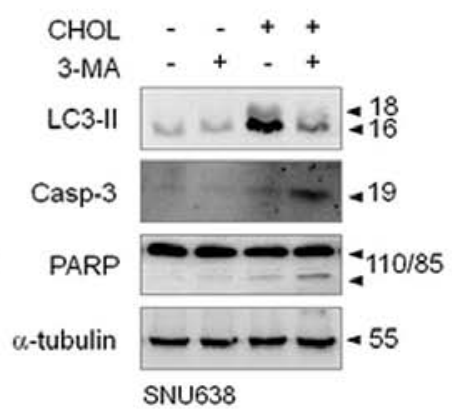

D
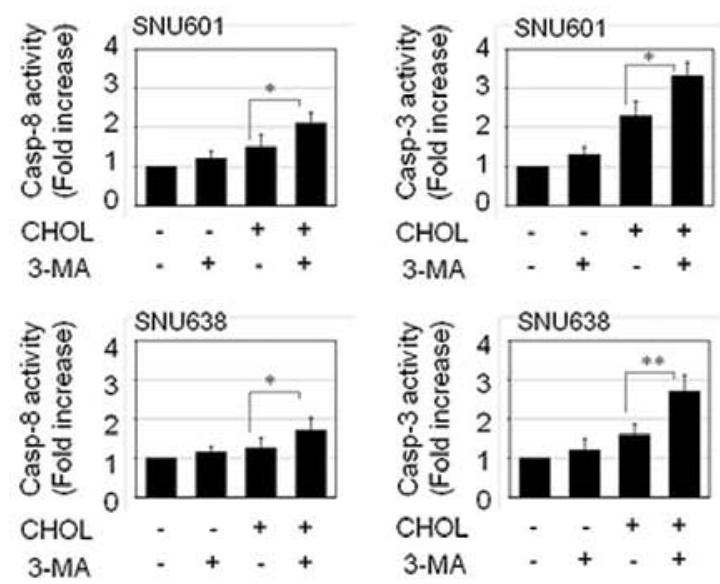

Figure 3. Cholesterol-induced autophagic cell death shifts to apoptosis by the autophagic inhibitor 3-MA. (A-D) Cells were exposed to 50 $\mu \mathrm{M}$ cholesterol for $48 \mathrm{~h}$ with or without $5 \mathrm{mM}$ of 3-MA and analyzed for (A) assessment of viability by MTT assay, (B) detection of apoptotic nuclei and autophagic vacuole by staining with Hoechst 33342 and MDC, respectively, (C) detection of LC3-II, active caspase-3, PARP and $\alpha$-tubulin by immunoblotting, and (D) measurement of caspase activity. ${ }^{*} \mathrm{p}<0.01,{ }^{* *} \mathrm{p}<0.005$.

cell lines was decreased by cholesterol, active apoptotic induction was observed only in SNU601 cells. Thus, we also attempted to determine whether other types of cell death are involved in cholesterol-induced reduction of cell viability. Because cleavage of LC3I to LC3II is a sign of autophagy, we examined the question of whether autophagy occurred in this event by detection of LC3II protein level. Treatment with cholesterol resulted in a mild increase in LC3II level in SNU601 cells, with a stronger increase in SNU638 and SNU216 cells (Fig. 2C). Furthermore, essential autophagic factor, ATG5 was also accumulated in SNU638 and SNU216 cells. However, necrotic features, PI inclusion or $\mathrm{LDH}$ release were not observed in these cell lines by $48 \mathrm{~h}$ (laboratory observation). Therefore, cholesterol seemed to trigger cell killing by apoptosis and autophagy in GC cells.

Inhibition of autophagy increases apoptosis. Autophagy may function as a protective mechanism and as a cell death mechanism. If this cholesterol-induced autophagy exerts a protective role, prevention of autophagy will further enhance cell death upon exposure to cholesterol. Nevertheless, inhibition of autophagy by 3-methyladenine (3-MA) did not aggravate cholesterol-mediated cell toxicity (Fig. 3A). This implies that cholesterol-induced autophagy is not a protective autophagy, but a process of a cell death. However, inhibition of autophagic death did not restore viability of cholesterol-treated cells either. Autophagy inhibitor, 3-MA resulted in a decrease in the number of autophagic vacuoles, as detected by MDC staining.
However, instead of a decrease of autophagic death, 3-MA led to an increase in apoptosis, as detected by elevated apoptotic body formation, cleavage of procaspase- 3 and PARP, and activation of caspase- 3 and caspase- 8 (Fig. 3B-D). Therefore, it appears that the fate of the cell has already been determined as death before decision of cell death mode between autophagic death and apoptosis; thus, blockade of the autophagic pathway may take a bypass to the apoptotic pathway instead of cell survival.

Transient inactivation of the ERK1/2 pathway is triggered by cholesterol. Mitogen-activated protein kinase (MAPK) family proteins play critical roles in regulation of cell survival and death; therefore, in order to determine the signaling mechanism involved in cholesterol-induced GC cell death, we first examined the effect of cholesterol on activities of MAPK family members. Exposure to cholesterol triggered an abrupt and transient decrease of ERK1/2 phosphorylation not affecting JNK or p38MAPK in both SNU601 and SNU638 cell lines. MEK1, upstream kinase of ERK1/2, was also transiently dephosphorylated by cholesterol (Fig. 4A). To examine the role of the ERK1/2 pathway in cholesterol-treated cells, we observed the effect of cholesterol in CA-MEK1 overexpressed cells. In comparison with control cells, CA-MEK1 expressed cells demonstrated significant rescue of cell viability (Fig. 4B). Thus, reduction of ERK activity appears to be responsible for cholesterol-mediated cell death. Nevertheless, because 
A

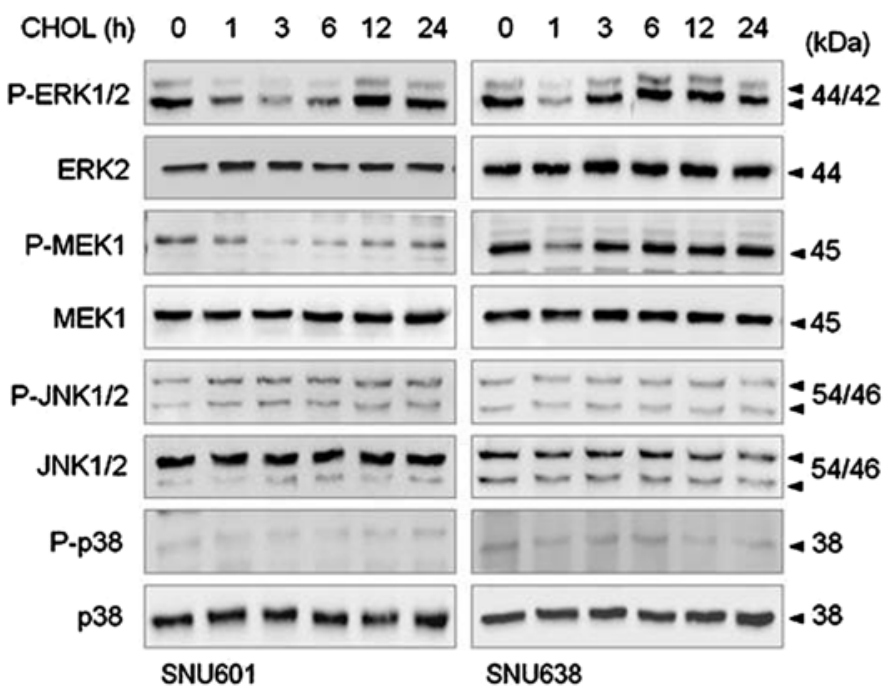

B
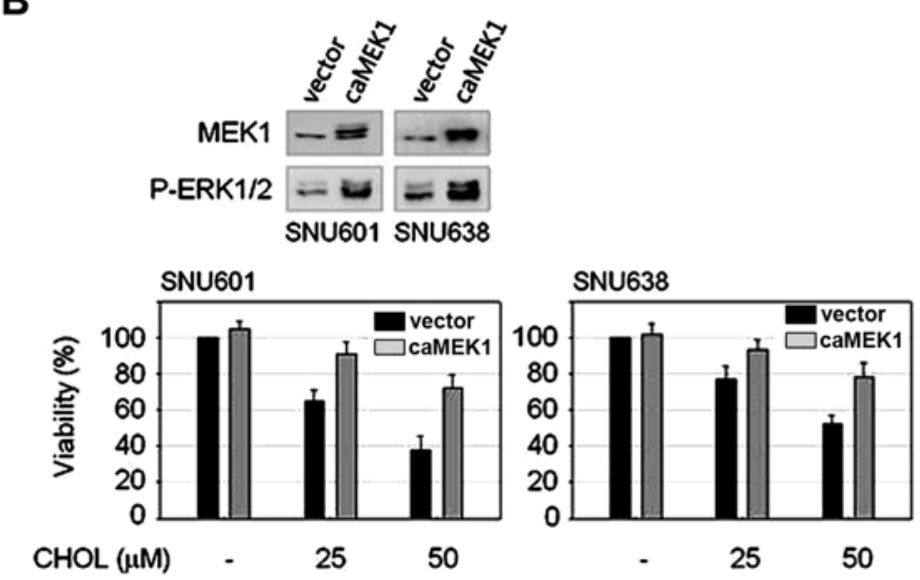

C
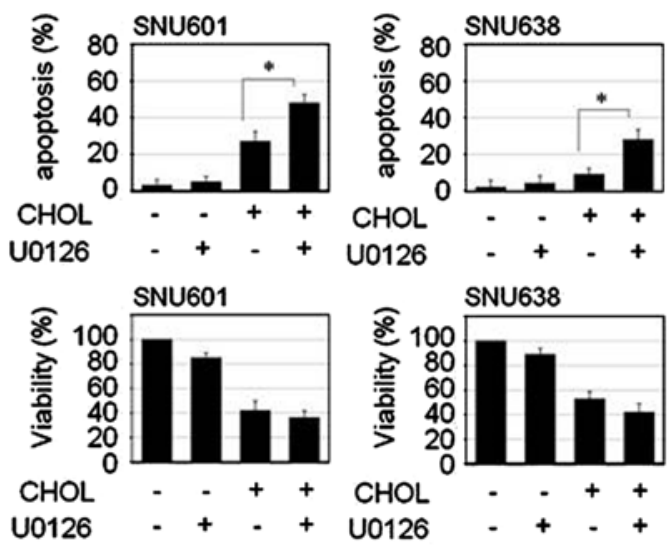

D

Figure 4. Cholesterol-triggered transient inactivation of ERK1/2 is responsible for reduction of GC cell viability. (A) SNU601 and SNU638 cells were exposed to $25 \mu \mathrm{M}$ cholesterol for the indicated times and analyzed by immunoblotting. (B) SNU601 and SNU638 cells were transfected with empty vector or CA-MEK1 overexpression vector and exposed to cholesterol for $48 \mathrm{~h}$; cell viability was then measured by MTT assay. (C and D) Cells were exposed to $50 \mu \mathrm{M}$ cholesterol in the absence or presence of U0126 for $48 \mathrm{~h}$ and (C) stained by Hoechst 33342 for measurement of apoptotic cells (upper panels) and analyzed by MTT assay for assessment of viability (lower panels), or (D) subjected to immunoblotting for detection of active caspase-3, PARP, LC3-II and $\alpha$-tubulin. ${ }^{*} \mathrm{p}<0.01$.

phospho-status of ERK was recovered soon after dephosphorylation upon treatment with cholesterol, we investigated the role of restored ERK activity. MEK inhibitor, U0126, was added to medium $3 \mathrm{~h}$ after treatment with cholesterol in order to inhibit reactivation of ERK1/2. Combination of U0126 resulted in strongly increased apoptosis compared to cholesterol treatment alone, as demonstrated by enhancement of apoptotic body formation and cleavage of procaspase-3 and PARP (Fig. 4C and D). However, the level of autophagic marker, LC3II, was not altered by U0126 in either cell type (Fig. 4D), indicating that inhibition of the late ERK pathway resulted in activation of strong apoptotic signaling. These results suggest that cholesterol-induced early downregulation of ERK signaling evokes loss of cell viability, and subsequent restoration of ERK activity may be responsible for inhibition of apoptotic pathway.

Membrane death receptor TRAIL-R2/DR5 is involved in cholesterol-mediated gastric cancer cell death. Cholesterol is an essential component of the cell membrane and choles- terol-enriched membrane lipid rafts can affect membrane receptor-linked signal regulation. Furthermore, in this study, treatment with cholesterol resulted in activation of caspase-8, a representative death receptor-mediated apoptotic enzyme. Thus, we attempted to determine whether the death receptor pathway is involved in this cholesterol-mediated cell death event. In immunoblot analysis to assess expression of death receptors; TRAIL-R1/DR4, TRAIL-R2/DR5 and Fas/CD95, increase of TRAIL-R2/DR5 protein was detected after treatment with cholesterol in both cell lines, however, protein levels of TRAIL-R1/DR4 and Fas/CD95 were unchanged by cholesterol or were too low to be observed in these cells (Fig. 5A). In order to understand the role of TRAIL-R2/DR5 overexpression in cholesterol-mediated apoptosis, we performed an interference assay using siRNA of TRAIL-R2/DR5. Compared to scrambled control RNA (CTL RNAi) transfection, TRAIL-R2/DR5 siRNA transfection resulted in a decrease in cholesterol-induced apoptosis in both GC cell lines (Fig. 5B). In addition, knockdown of TRAIL-R2/DR5 reduced not only 

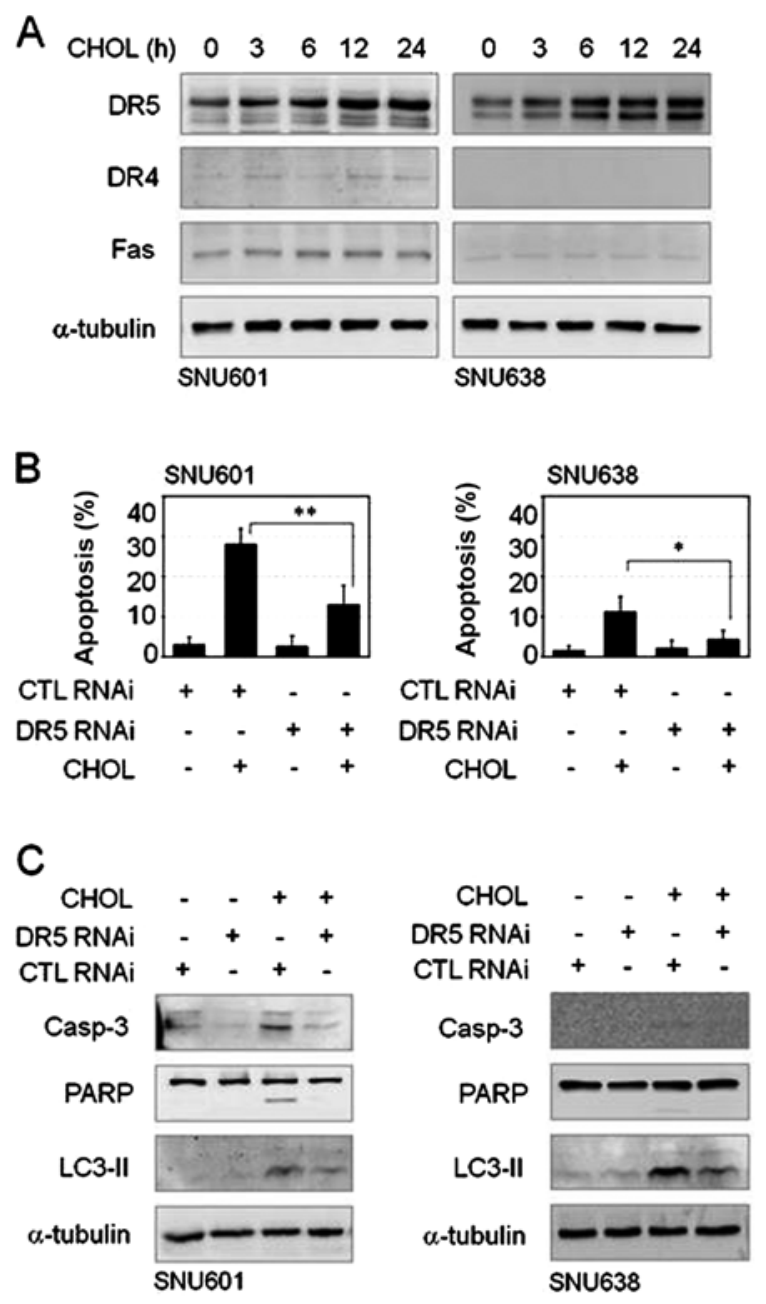

Figure 5. Membrane death receptors are involved in cholesterol-induced cell death in GC cells. (A) Cells were exposed to $50 \mu \mathrm{M}$ cholesterol for the indicated times and analyzed by immunoblotting for detection of membrane death receptors DR4, DR5 and Fas. (B and C) Cells transfected with scrambled siRNA (CTL RNAi) or DR5 siRNA were incubated with $50 \mu \mathrm{M}$ cholesterol for $48 \mathrm{~h}$ and analyzed by Hoechst 33342 staining for (B) detection of apoptotic body formation, or (C) subjected to immunoblotting for detection of active caspase-3, PARP and LC3-II. ${ }^{*} \mathrm{p}<0.01,{ }^{* *} \mathrm{p}<0.005$.

cleavage of caspase-3 and PARP but also elevation of LC3II level (Fig. 5C). Thus, TRAIL-R2/DR5 appeared to be linked to apoptotic and autophagic death pathways in response to cholesterol.

\section{Discussion}

Cholesterol plays an important role in the human body as a precursor of critical biochemical molecules, including steroid hormones, vitamin D and bile acids. In addition, cholesterol is an essential component enriched in biological membranes and participates in control of cellular membrane fluidity and lipid rafts. Lipid rafts serve as signaling platforms at the cell membrane and modification of major lipid raft components, such as cholesterol, sphingolipid and ceramide, play a role in regulation of cell viability under cytotoxic stimuli (19). Mediation of akt-regulated cell survival by cholesterol-rich lipid rafts and increased apoptosis by depletion of cholesterol in prostate cancer cells have been reported $(20,21)$. In addition, intake of high cholesterol has been reported to show correlation with an increased risk of occurrence of certain cancers, including breast, prostate, and colon cancer. However, low level of cholesterol may also be harmful in certain cases. Cholesterol depletion prohibits the effect of chemotherapeutics by inhibition of membrane raft formation, because certain types of chemotherapeutic drugs require lipid raft-dependent death receptor activation for induction of cancer cell death $(22,23)$. In addition, several epidemiological studies have reported an association of serum cholesterol levels at the lower end of the distribution with risk of cancer mortality (10,24-27). In this study, increased level of cholesterol led to markedly reduced viability and clonogenicity of stomach cancer cells. Increased level of cholesterol was previously shown to induce apoptosis in macrophages through various mechanisms involving the Fas pathway, the mitochondrial apoptotic pathway and the endoplasmic reticulum response (28-30). In measurement of morphological and biochemical features induced by cholesterol, both apoptotic and autophagic deaths were observed in GC cells, although dominant type of death was cell line specific. Cholesterol stimulated accumulation of ATG5 and cleavage of LC3 with a very low level of apoptotic body formation in SNU638 and SNU216 cells, indicating that autophagic death occurred mainly in these cells. However, in SNU601 cells, ATG5 was degraded and LC3 cleavage was mild, instead, strong apoptotic features, including nuclear fragmentation, caspase-3 cleavage and PARP degradation were detected.

Of particular interest, crosstalk appeared to occur between the apoptotic pathway and autophagic pathway. In combination of cholesterol with autophagy inhibitor 3-MA, autophagic death was reduced and resulted in switching the death mode to apoptosis without significant effect on cell viability. Early dephosphorylation of ERK appeared to be responsible for the cholesterol-mediated cell death since overexpression of CA-MEK1 resulted in significantly restored cell viability. A few hours after dephosphorylation of ERK, subsequent rephosphorylation was observed and suppression of ERK reactivation by post-treatment with MEK inhibitor U0126 resulted in elevation of apoptosis and decrease of cell viability, indicating that this restoration of ERK activity is mainly involved in the anti-apoptotic pathway. We also demonstrated that exposure of GC cells to cholesterol increased expression of TRAIL-R2/DR5 protein and this was involved in cholesterol-induced GC cell death. Knockdown of TRAIL-R2/DR5 resulted in reduced activation of caspase-3, cleavage of PARP and LC3II level in response to cholesterol, thus, TRAIL-R2/DR5 appeared to be linked to both apoptotic and autophagic death signaling. To understand the relationship between ERK pathway and TRAIL-R2/DR5 induction, we measured cholesterol-mediated ERK phosphorylation in a condition of TRAIL-R2/DR5 interference, and knockdown of TRAIL-R2/DR5 had no effect on reduction of ERK phosphorylation, and inhibition of ERK reactivation by U0126 did not enhance TRAIL-R2/DR5 induction (data not shown). Thus, these two events seem to be independently controlled by cholesterol in GC cells.

Based on these results, our findings demonstrated that exposure of GC cells to cholesterol resulted in stimulation of apoptotic and autophagic death through inactivation of ERK 
and induction of TRAIL-R2/DR5, and this may be one of the explanations that serum cholesterol levels at the lower end of the distribution are related to higher risk of stomach cancer mortality.

\section{Acknowledgements}

This research was supported by the Basic Science Research Program through the National Research Foundation of Korea (NRF) funded by the Ministry of Education, Science and Technology (NRF-2011-0014540).

\section{References}

1. Greenlee RT, Hill-Harmon MB, Murray T and Thun M: Cancer statistics, 2001. CA Cancer J Clin 51: 15-36, 2001.

2. Roukos DH: Current status and future perspectives in gastric cancer management. Cancer Treat Rev 26: 243-255, 2000.

3. Fiedorek SC, Malaty HM, Evans DL, Pumphrey CL, Casteel HB, Evans DJ Jr and Graham DY: Factors influencing the epidemiology of Helicobacter pylori infection in children. Pediatrics 88: 578-582, 1991.

4. Plummer M, Franceschi S and Munoz N: Epidemiology of gastric cancer. IARC Sci Publ 2004: 311-326, 2004

5. Lunet N, Valbuena C, Vieira AL, Lopes C, Lopes C, David L, Carneiro $\mathrm{F}$ and Barros $\mathrm{H}$ : Fruit and vegetable consumption and gastric cancer by location and histological type: case-control and meta-analysis. Eur J Cancer Prev 16: 312-327, 2007.

6. Kaaks R, Tuyns AJ, Haelterman M and Riboli E: Nutrient intake patterns and gastric cancer risk: a case-control study in Belgium. Int J Cancer 78: 415-420, 1998.

7. Palli D, Russo A and Decarli A: Dietary patterns, nutrient intake and gastric cancer in a high-risk area of Italy. Cancer Causes Control 12: 163-172, 2001.

8. De Stefani E, Correa P, Boffetta P, Deneo-Pellegrini H, Ronco AL and Mendilaharsu M: Dietary patterns and risk of gastric cancer: a case-control study in Uruguay. Gastric Cancer 7: 211-220, 2004

9. Asano K, Kubo M, Yonemoto K, Doi Y, Ninomiya T, Tanizaki Y, Arima H, Shirota T, Matsumoto T, Iida M and Kiyohara Y: Impact of serum total cholesterol on the incidence of gastric cancer in a population-based prospective study: the Hisayama study. Int J Cancer 122: 909-914, 2008

10. Dessi S, Batetta B, Pulisci D, Spano O, Anchisi C, Tessitore L, Costelli P, Baccino FM, Aroasio E and Pani P: Cholesterol content in tumor tissues is inversely associated with highdensity lipoprotein cholesterol in serum in patients with gastrointestinal cancer. Cancer 73: 253-258, 1994.

11. Jafri H, Alsheikh-Ali AA and Karas RH: Baseline and on-treatment high-density lipoprotein cholesterol and the risk of cancer in randomized controlled trials of lipid-altering therapy. J Am Coll Cardiol 55: 2846-2854.

12. Levy RI: Cholesterol and disease - what are the facts? JAMA 248: 2888-2890, 1982.

13. Eichholzer M, Stahelin HB, Gutzwiller F, Ludin E and Bernasconi F: Association of low plasma cholesterol with mortality for cancer at various sites in men: 17-y follow-up of the prospective Basel study. Am J Clin Nutr 71: 569-574, 2000.
14. Kritchevsky SB and Kritchevsky D: Serum cholesterol and cancer risk: an epidemiologic perspective. Annu Rev Nutr 12: 391-416, 1992.

15. Schatzkin A, Hoover RN, Taylor PR, Ziegler RG, Carter CL, Larson DB and Licitra LM: Serum cholesterol and cancer in the NHANES I epidemiologic follow-up study. National Health and Nutrition Examination Survey. Lancet 2: 298-301, 1987.

16. Tornberg SA, Holm LE, Carstensen JM and Eklund GA: Cancer incidence and cancer mortality in relation to serum cholesterol. J Natl Cancer Inst 81: 1917-1921, 1989.

17. Wannamethee G, Shaper AG, Whincup PH and Walker M: Low serum total cholesterol concentrations and mortality in middle aged British men. BMJ 311: 409-413, 1995.

18. Brunet A, Pages G and Pouyssegur J: Constitutively active mutants of MAP kinase kinase (MEK1) induce growth factor-relaxation and oncogenicity when expressed in fibroblasts. Oncogene 9: 3379-3387, 1994.

19. Edidin M: The state of lipid rafts: from model membranes to cells. Annu Rev Biophys Biomol Struct 32: 257-283, 2003.

20. Zhuang L, Kim J, Adam RM, Solomon KR and Freeman MR: Cholesterol targeting alters lipid raft composition and cell survival in prostate cancer cells and xenografts. J Clin Invest 115: 959-968, 2005

21. Zhuang L, Lin J, Lu ML, Solomon KR and Freeman MR Cholesterol-rich lipid rafts mediate akt-regulated survival in prostate cancer cells. Cancer Res 62: 2227-2231, 2002.

22. Gajate C, Gonzalez-Camacho F and Mollinedo F: Involvement of raft aggregates enriched in Fas/CD95 death-inducing signaling complex in the antileukemic action of edelfosine in Jurkat cells. PLoS One 4: e5044, 2009.

23. Mollinedo F and Gajate C: Fas/CD95 death receptor and lipid rafts: new targets for apoptosis-directed cancer therapy. Drug Resist Updat 9: 51-73, 2006.

24. Iribarren C, Reed DM, Chen R, Yano K and Dwyer JH: Low serum cholesterol and mortality. Which is the cause and which is the effect? Circulation 92: 2396-2403, 1995.

25. Kitahara CM, Berrington de Gonzalez A, Freedman ND, Huxley R, Mok Y, Jee SH and Samet JM: Total cholesterol and cancer risk in a large prospective study in Korea. J Clin Oncol 29: $1592-1598$.

26. Schuit AJ, Van Dijk CE, Dekker JM, Schouten EG and Kok FJ: Inverse association between serum total cholesterol and cancer mortality in Dutch civil servants. Am J Epidemiol 137: 966-976, 1993.

27. Tornberg SA, Carstensen JM and Holm LE: Risk of stomach cancer in association with serum cholesterol and beta-lipoprotein. Acta Oncol 27: 39-42, 1988.

28. Tabas I: Consequences of cellular cholesterol accumulation: basic concepts and physiological implications. J Clin Invest 110: 905-911, 2002.

29. Yao PM and Tabas I: Free cholesterol loading of macrophages induces apoptosis involving the fas pathway. J Biol Chem 275: 23807-23813, 2000

30. Yao PM and Tabas I: Free cholesterol loading of macrophages is associated with widespread mitochondrial dysfunction and activation of the mitochondrial apoptosis pathway. J Biol Chem 276: 42468-42476, 2001. 